\title{
Background rejection and sensitivity for new generation Ge detectors experiments
}

\author{
H. Gómez*, S. Cebrián, J.A. Villar. \\ Laboratorio de Física Nuclear y Astropartículas. Universidad de Zaragoza. \\ E-mail: hgomez@unizar.es
}

\begin{abstract}
Germanium detectors have been used in several experiments searching for Rare Events due to their good features (detection efficiency, energy resolution or robustness). New generation detectors, like broad energy detectors or segmented ones, could improve the sensitivity of this kind of experiments since different background rejection techniques could be developed without losing detection efficiency. Within these techniques, analysis of the pulse shapes in a segmented detector, in the segment where the energy deposit happens and in the adjacent ones (the so-called net and induced signals respectively), seems to be one of the most powerful ones. Applying to ${ }^{76} \mathrm{Ge}$ double beta decay region of interest (2.0-2.1 MeV), a set of routines for pulse generation and analysis has allowed to estimate that the background could be reduced by a factor 25 , keeping a detection efficiency for neutrinoless double beta decay events of $76.3 \%$ using $2 \mathrm{~kg}$ detectors, which implies that exploration of the inverted hierarchy region of neutrino masses could be reachable. A summary of the development of pulse generation and analysis routines (both for net and induced signals) will be given, showing the rejection factors and detection efficiency values obtained for the different configurations considered.
\end{abstract}

Identification of Dark Matter 2010

July 26 - 302010

University of Montpellier 2, Montpellier, France

\footnotetext{
*Speaker.
} 


\section{Introduction}

Several experiments in the Rare Event searches field, have considered the utilization of new generation germanium detectors, like broad energy or segmented ones, due to the excellent features they offer (radiopurity, high detection efficiency and energy resolution values or robustness). For example Edelweiss or CDMS collaborations use cryogenic germanium detectors looking for Dark mater, while Gerda or Majorana experiments plan to use broad-energy [1, 2] or segmented [3, 4, 5] detectors, enriched in ${ }^{76} \mathrm{Ge}$, for the study of the neutrinoless double beta decay $(0 v \beta \beta)$ of this isotope.

Focused on the neutrinoless double beta decay, the so-called new generation experiments aim to explore the inverted hierarchy of the neutrino masses, that corresponds to a neutrino effective mass up to $\sim 50 \mathrm{meV}$ [6]. To reach the sensitivity needed to explore this region, the experiments must have high detection efficiency values for $0 v \beta \beta$ keeping low background levels in the energy region of interest (around the transition energy $Q_{\beta \beta}$ ). This low background level is reachable by the combination of different issues: selection of radiopure materials for the experimental setup construction, installation of active and passive shielding, placement of the experiment underground and the development of background events rejection techniques are probably the most important ones.

Previous studies revealed that the analysis of the pulse shape generated in segmented germanium detectors with three-dimensional resolution, seems to be one of the most powerful tools to identify background events, for further rejection, keeping high detection efficiency for $0 v \beta \beta$ events [7]. Trying to estimate this background rejection capability, a set of simulation of events and further pulse generation and analysis algorithms was developed. A brief description of these simulations and algorithms and the results obtained are presented here, estimating the sensitivity that could be reached by a $0 v \beta \beta$ experiment using enriched germanium detectors and this technique. The performance of novel pulse shape analysis techniques has been also studied in other works $[8,9,10,11]$.

\section{Setup}

Simulation of background and signal events is the first step to carry out the pulse generation and analysis task. The most important issues to take into account are the definition of the experimental setup, paying special attention to the geometry and materials trying to define it as similar as the real one, and the definition of the simulated events, since a realistic background model will provide more accurate estimations about the background rejection capability of the pulse shape analysis (PSA).

\subsection{Geometry and materials}

$0 v \beta \beta$ experiments using germanium detectors enriched in ${ }^{76} \mathrm{Ge}$ isotope, expects to operate an array of these crystals trying to have enough emitter mass (about hundreds of $\mathrm{kg}$ ) to reach the expected sensitivity. In addition, the use of several detectors allows the application of anticoincidence techniques between different crystals. For this work, only one detector has been defined 
(anticoincidence between detectors is not studied), but versatile enough to allow further changes. The setup is composed by three main parts:

- A natural germanium crystal from 0.1 to $4 \mathrm{~kg}$, to study the dependance between background level and detector mass, and cylindrical shape, having the same height and diameter.

- Copper cryostat, closing the germanium crystal. It is based on IGEX experiment design and it is composed by three different parts, with dimensions dependant on the crystal size.

- Experimental place, surrounding the detector. It is defined as $2 \mathrm{~m}$ diameter sphere, being big enough for further extension of the experimental setup. The volume inside this sphere is defined as air.

\subsection{Simulated events}

The transition energy $\left(Q_{\beta \beta}\right)$ for ${ }^{76} \mathrm{Ge}$ is $2039.5 \mathrm{keV}$, which fix the energy region of interest for $0 v \beta \beta$ experiments between 2.0-2.1 MeV. The simulated events have been chosen to be the typical contaminations present at the setup materials or from the environmental background that have influence in this region of interest, that are:

- ${ }^{60} \mathrm{Co}$ and ${ }^{68} \mathrm{Ge}$ contaminations coming from the crystal due to the germanium cosmogenic activation.

- ${ }^{60} \mathrm{Co}$ contamination coming from the cryostat due to the intrinsic contamination of copper and cosmogenic activation.

- External ${ }^{208} \mathrm{Tl}$ and ${ }^{214} \mathrm{Bi}$ emitted from the experimental place as main contributions of the environmental background.

- $2 v \beta \beta$ events of ${ }^{76} \mathrm{Ge}$ emitted form the crystal with an energy higher than $2.0 \mathrm{MeV}$.

All these events represent around 95\% of the total background in the region of interest (only alpha events, neutrons and muons are not considered). In order to estimate the signal detection efficiency of the PSA, $0 v \beta \beta$ events emitted form the crystal were also simulated. To make all the simulations GEANT4 package was used, although the generation of $\beta \beta$ events was made using DECAY0, generating in all the cases enough number of events to have an statistical error below $3 \%$. All the simulated events were validated before using it for the pulse generation and analysis by checking the energy spectra they produce or the correlation between energy and angle of emission in the case of $\beta \beta$ events.

\section{Pulse generation}

As it was mentioned before, PSA with three-dimensional reoslution (3D-PSA) is only possible if segmented crystals are considered, and pulses generated in the segment where interaction happens (net signal) and in the adjacent ones (induced signals) must be analyzed. For all the signals, the pulse is generated as the representation of charge variation during time, that is represented by Eq. $3.1[12]$ : 

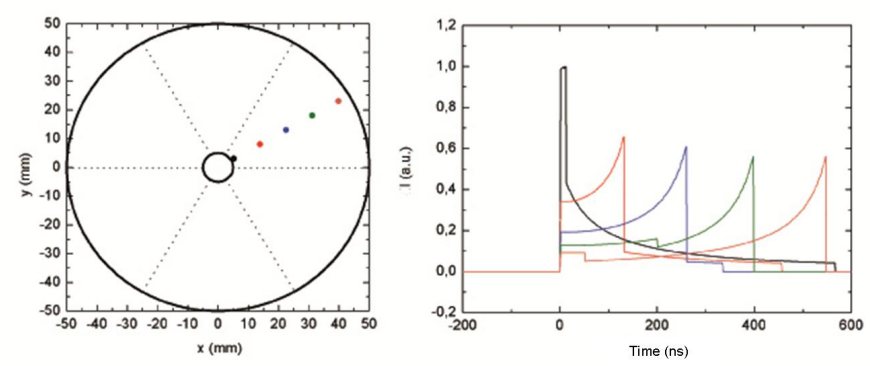

Figure 1: Examples of different ideal net signals (right) from the corresponding energy deposit position (left).

$$
\frac{d Q}{d t}=\frac{q_{i} \mu_{i} \vec{E}(\vec{r}) \vec{E}_{w}(\vec{r})}{V_{0}\left(1+\left(\frac{|\vec{E}(\vec{r})|}{E_{s a t}}\right)^{\beta}\right)^{1 / \beta}}
$$

which depends on the voltage applied to the detector $\left(V_{0}\right)$, the electric field produced inside the crystal due to this voltage $(\vec{E}(\vec{r}))$ and the denominated weighting field $\left(\vec{E}_{w}(\vec{r})\right)$, a mathematical formalism that represents the electric field in a multielectrode system (like a segmented detector) when all the electrodes have null potential except one. $\mu, E_{\text {sat }}$ and $\beta$ are parameters characterizing the velocity of charge carriers. $\vec{E}_{w}(\vec{r})$ corresponds to $\vec{E}(\vec{r})$ when the net signal is generated but is different for the induced ones. To calculate both $\vec{E}(\vec{r})$ and $\vec{E}_{w}(\vec{r})$ for the pulses generation, a finite element calculation software was used.

Following this generation method different features for net and induced signals are observed:

- Net signal.

These pulses have 2 singular points corresponding to the holes and electrons arrival to the electrodes. The time position of these points depends on the radial coordinate of the energy deposit (Fig. 1). For this reason, the analysis of the net signals based on the position of the singular points, only provide radial sensitivity about the position of the energy deposit.

- Induced signal.

Signals produced in the adjacent segments have not any new temporal information with respect to the net signal (Fig. 2). On the other hand they have some common features, they are bipolar signals with null net area and absolute area $\left(A_{A}\right.$, sum of the absolute values of the areas of the positive and negative part of the pulse) lower than the area of the corresponding net signal. This $A_{A}$ is the most interesting value for further analysis of these signals.

An important issue for pulse generation, specially for net signals, is the denominated characteristic time. Electronics used to readout the signals could distort them, making more difficult the analysis. This effect has been taken into account by convoluting the obtained signals (ideal) with a transfer function with an arbitrary time constant, generating a more realistic signal. The study of the PSA efficiency to discriminate background events for different time constant values, will fix the optimal one. 


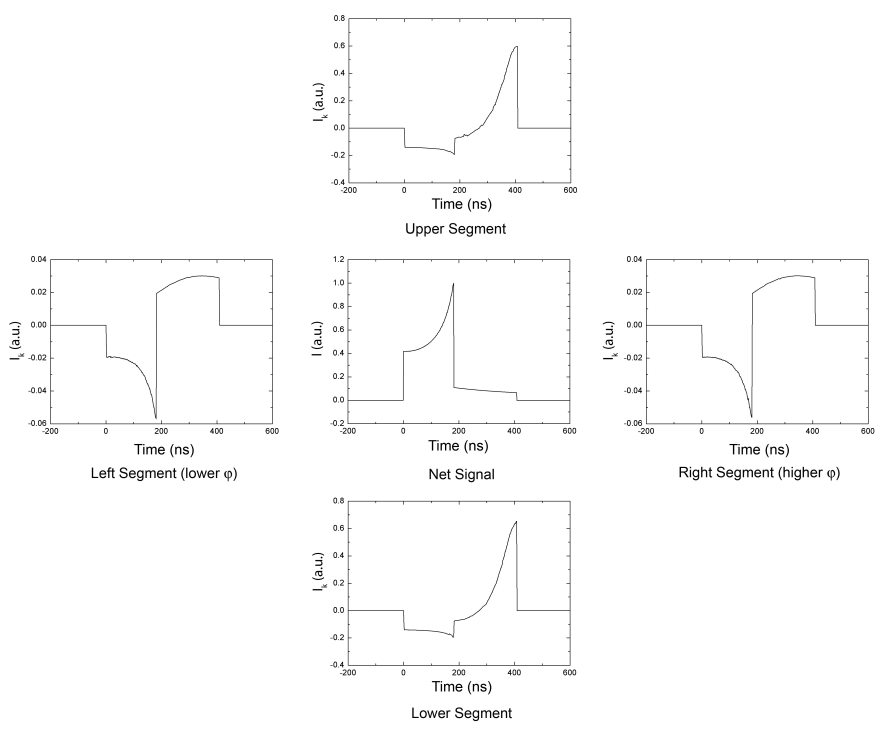

Figure 2: Example of an ideal net signal and its corresponding induced ones for an event with only one energy deposit.

\section{Pulse analysis}

3D-PSA can discriminate signal events from background ones based on the fact that signal events release all the energy in a very small region of the detector that could be considered like punctual (monosite event). On the other hand, background events are mostly produced by different interactions separated in the space, which means that the event is a group of energy deposits (multisite event). For this reason it is not necessary to locate where the energy deposit happens, only the identification of the number of energy deposits per event is required. Since there are differences between net and induced signals, the analysis methods applied to each of them should be also different for each of these signals.

The net signal analysis method is based on the property that each energy deposit produces 2 singular points, which correspond to relative maxima in the pulse. If the derivative has 2 maxima, the event is considered monosite. If 3 or more maxima are located, the event is multisite. As it was mentioned before, the maxima identification depends on the time constant considered for the convolution of the ideal pulse.

The induced signal analysis is realized comparing the $A_{A}$ of the studied pulses with the same parameter of the corresponding pulses of an event with the same energy but with only one energy deposit placed in the center of energy (expected position of the energy deposit if the event was considered as monosite). It was checked that ratio of $A_{A}$ of the analyzed signals and the corresponding Center of Energy ( $\mathrm{CoE})$ one is bigger for multisite events than for monosite ones, for which it should be the unit. This analysis method is independent of the time constant considered, since the transfer function has been defined with unitary modulus and does not vary the area of the signals after the convolution. To develop this analysis method, CoE events library was generated. 
Table 1: Summary of background level of the different contributions considered $\left(10^{-3} \mathrm{c} / \mathrm{keV} / \mathrm{kg} / \mathrm{y}\right)$ and detection efficiency values for $0 v \beta \beta$ events, for a $2 \mathrm{~kg}$ crystal with $6 \times 9$ segmentation scheme and $4 \mathrm{~kg}$ crystal with $6 \times 11$, before applying any PSA, after analyzing net signal with $40 \mathrm{~ns}$ characteristic time, and after analyzing net and induced signals. Initial background conditions have been taken from [7].

\begin{tabular}{|c|c|c|c|c|c|c|}
\hline & \multicolumn{3}{|c|}{$2 \mathrm{~kg}$} & \multicolumn{3}{|c|}{$4 \mathrm{~kg}$} \\
\hline $\begin{array}{l}\text { Background } \\
\text { source }\end{array}$ & Raw & $\begin{array}{l}\text { Net Signal } \\
\text { 40ns }\end{array}$ & $\begin{array}{c}\text { Induced } \\
\text { Signal }\end{array}$ & Raw & $\begin{array}{l}\text { Net Signal } \\
\text { 40ns }\end{array}$ & $\begin{array}{c}\text { Induced } \\
\text { Signal }\end{array}$ \\
\hline $\begin{array}{c}\text { Internal }{ }^{60} \mathrm{Co} \\
5 \mathrm{~kg}^{-1} \mathrm{~d}^{-1} ; 30 \mathrm{~d} \text { exp.; } 0 \mathrm{~d} \text { cool. }\end{array}$ & 2.90 & 0.01 & $<0.01$ & 3.73 & 0.01 & $<0.01$ \\
\hline $\begin{array}{c}\text { Internal }{ }^{68} \mathrm{Ge} \\
1 \mathrm{~kg}^{-1} \mathrm{~d}^{-1} ; 180 \mathrm{~d} \text { exp } ; 180 \mathrm{~d} \text { cool. }\end{array}$ & 12.50 & 0.26 & 0.24 & 38.66 & 0.23 & 0.20 \\
\hline $\begin{array}{c}\text { External }{ }^{208} \mathrm{Tl} \\
\phi \sim 0.1 \mathrm{\gamma cm}^{-2} \mathrm{~s}^{-1}\end{array}$ & 0.38 & 0.16 & 0.16 & 0.30 & 0.11 & 0.11 \\
\hline $\begin{array}{l}\text { External }{ }^{214} \mathrm{Bi} \\
\phi \sim 0.38 \mathrm{\gamma cm}^{-2} \mathrm{~s}^{-1}\end{array}$ & 0.17 & 0.08 & 0.08 & 0.14 & 0.07 & 0.07 \\
\hline $\begin{array}{c}\text { Internal }{ }^{232} \mathrm{Th} \text { in lead } \\
1 \mu B q / k g\end{array}$ & 2.82 & 1.23 & 1.21 & 2.25 & 0.88 & 0.86 \\
\hline $\begin{array}{c}{ }^{60} \mathrm{Co} \text { from } \mathrm{Cu} \text { cryostat } \\
1 \mathrm{mBq} / \mathrm{kg}\end{array}$ & 28.47 & 0.15 & 0.14 & 33.67 & 0.27 & 0.24 \\
\hline $\begin{array}{l}2 v \beta \beta \text { events } \\
1.2110^{-4} \mathrm{~kg}^{-1} y^{-1} \\
\end{array}$ & 0.09 & 0.08 & 0.08 & 0.09 & 0.08 & 0.08 \\
\hline TOTAL & 47.33 & 1.97 & 1.91 & 78.84 & 1.65 & 1.56 \\
\hline $\begin{array}{c}0 v \beta \beta \text { events } \\
\text { detection efficiency }\end{array}$ & & 76.66 & 76.28 & & 76.74 & 75.59 \\
\hline
\end{tabular}

\section{Results}

The pulse generation and analysis was carried out using 2 and $4 \mathrm{~kg}$ crystals with $6 \times 9$ and $6 \times 11$ segmentation schemes respectively (i.e. with 6 longitudinal segments and 9 and 11 transversal ones to have 54 and 66 segments respectively), which are more suitable to reach higher sensitivity in a $0 v \beta \beta$ experiment. First step was the analysis of net signals using different time constant values. Combination of detection efficiency and background level reached fixes the optimal value of the time constant in $40 \mathrm{~ns}$. With this value induced signal analysis was done. Table 1 summarizes the background level and detection efficiency reachable after net and induced signal analysis for both crystals considered.

New generation $0 v \beta \beta$ experiments using germanium detectors (with several hundreds of ${ }^{76} \mathrm{Ge}$ ), expecting to explore the inverted hierarchy of neutrino masses, need to reach a background level around $10^{-3} \mathrm{c} / \mathrm{keV} / \mathrm{kg} / \mathrm{y}$, keeping a detection efficiency for signal events around $75 \%$. Table 1 shows that these values are almost reachable applying PSA for 2 and $4 \mathrm{~kg}$ detectors.

With these values, an effective neutrino mass region between 39 and $56 \mathrm{meV}$ (depending on the nuclear matrix elements considered and taking standard energy resolution for germanium detectors) 
could be explored using $2 \mathrm{~kg}$ segmented crystals with an exposure of $1000 \mathrm{~kg} \times \mathrm{y}$. For $4 \mathrm{~kg}$ ones, the region is between 37 and $53 \mathrm{meV}$.

\section{Summary and Conclusions}

Germanium detectors are one of the best options for Rare Event searches due to its excellent features. Specially these detectors enriched in ${ }^{76} \mathrm{Ge}$ isotope are really suitable for $0 v \beta \beta$ experiments. PSA in segmented detectors seems to be one of the most powerful tools for background rejection, keeping high detection efficiency values, which implies an improvement on the sensitivity of the experiment.

For these reasons, a set of simulation and pulse generation and analysis algorithms were developed in order to quantify the background rejection capability, detection efficiency, and in consequence the expected sensitivity of an experiment using this technique. Results obtained revealed that exploring the inverted hierarchy of the neutrino masses (with a neutrino effective mass up to $\sim 50$ $\mathrm{meV}$ ) seems possible using 2 or $4 \mathrm{~kg}$ detectors with exposures around $1000 \mathrm{~kg} \times \mathrm{y}$, which are considered in new generation experiments.

\section{Acknowledgments}

This work has been supported by the Spanish Ministerio de Ciencia e Innovación (MICINN) FPA2008-03228, the CPAN project Ref. CSD2007-00042 from the Consolider-Ingenio 2010 program and the ILIAS integrating activity (Contract number: EU-RII3-CT2003-506222).

\section{References}

[1] P. S. Barbeau et al, JCAP 09 (2007)007.

[2] D. Budjas et al, J. of Instrum. 4 (2009) P10007.

[3] S. R. Elliot et al, Nucl. Instrum. Meth. A 558 (2006) 504.

[4] I. Abt et al, Nucl. Instrum. Meth. A 577 (2007) 574.

[5] B. Majorovits, Prog. Part. Nucl. Phys. 64 (2010) 264.

[6] F. T. Avignone III et al , Rev. Mod. Phys. 80 (2008) 481.

[7] H. Gómez et al, Astropart. Phys. 28 (2007) 435.

[8] I. Abt et al, Nucl. Instrum. Meth. A 570 (2007) 479.

[9] I. Abt et al, Eur. Phys. J. C 52 (2007) 19.

[10] D. B. Campbell et al, Nucl. Instrum. Meth. A 587 (2008) 60.

[11] I. Abt et al, Eur. Phys. J. C 68 (2010) 609.

[12] Th. Kroll, D. Bazzacco, Nucl. Instrum. Meth. A 463 (2001) 227. 